\title{
Stress and psychological resilience in intervention and rescue activities
}

\author{
Izabella Kovacs $^{1 *}$, Andrei-Lucian Gireadă ${ }^{1}$, Lorand Toth ${ }^{1}$, and Sorin Simion ${ }^{1}$ \\ ${ }^{1}$ National Institute for Research and Development in Mine Safety and Protection to Explosion - \\ INSEMEX Petroşani, 32-34 G-ral Vasile Milea Street, Petroşani 332047, Romania
}

\begin{abstract}
Nature of intervention and rescue personnel activity places them at the top of professions that face a considerable number of occupational health and safety risks. Often, this occupational category does not face a single safety risk, but a complex combination of risk factors, including unpredictability of situations in which they are required to work. Emergence of stress and other psychosocial risks in work processes requires the implementation of an anticipatory attitude and a constant level of vigilance to identify and evaluate them. In intervention and rescue, the issue of regulating and self-regulating the individual's behaviour is essential, as exceptional acts performed in unusual conditions require adaptive mechanisms as close as possible to perfection. Developing resilience should focus on amplifying already present strengths (physical and mental characteristics and abilities), rather than managing negative effects of operational stressors. The current paper presents a theoretical approach of the concept of resilience, appliable to intervention and rescue activities and suggests several ways to develop rescuer's resilience.
\end{abstract}

\section{Introduction}

Structure of intervention and rescue activity has two closely related parts: the objective part, represented by goals and tasks, which must be solved at certain qualitative and quantitative standards, and the subjective part represented by all abilities, capacities and resources employed by a person engaged in this activity.

Psychological selection/examination represent an important first step for an efficient process of work management. Psychological selection of personnel should focus on both individual psychological diagnosis and individual prognosis, revealing qualities and occupational contraindications, based on which an individual gets the recommendation of being or not being suited for a certain job/position.

Organizational environments have always been receptive to what psychologists call individual differences, such as asymmetries that underlie differences between two or more people. Employers are interested in knowledge regarding the occupation (skills, information that may be translated into abilities etc.), but also in mental attributes of their employees. Differences between individuals, in attributes such as intelligence, personality, knowledge are important to explain a wide range of results, including: academic, managerial,

* Corresponding author: izabella.eisler@insemex.ro 
professional achievements, communication with others, reactions in stressful situations, coping with stress etc.

A traumatic event has the potential to have negative consequences on a person, consequences that can be emotional, cognitive and / or behavioural. At the same time, however, it can cause the person to re-evaluate their own existence, to redefine the options they have, to reprioritize their life, cognitive processing that can lead to post-traumatic personal development.

Distinctions must also be made between post-traumatic growth and the concepts of resilience, robustness, optimism and a sense of coherence. All of these concepts describe certain personality traits that allow individuals to better manage traumatic events. Posttraumatic growth involves a qualitative transformation or change in the functioning of the individual, as opposed to seemingly similar concepts of resilience, a sense of coherence, optimism and robustness.

The philosopher Epictetus once wrote that man is disturbed, not by things, but by the opinions one has about things, Buddha stated that our lives are a creation of our mind, Shakespeare said that there is nothing, nor good, nor bad, only thinking makes them so and Milton says that the mind is a place in itself, and can make heaven from hell and hell from heaven. What do all these statements have in common? The answer is that most of the stress in an individual's life comes from the way one looks at and interprets his reality. The meanings that one attributes to things are the essential determinants of happiness and even professional and personal effectiveness.

Some studies show that improving psychological characteristics, such as a sense of coherence, resilience and the ability to psychologically adapt, can facilitate coping with difficult, traumatic situations $[1,2]$. Resilience refers to the ability to use available psychological, social, cultural and physical resources to support well-being, and also the ability to obtain these resources. At work. these characteristics can be improved through team cohesion, collective identity, common values, good leadership, trust and mission objectives (goal orientation).

The current paper is part of a broader doctoral studies research, aiming at developing a psychological training program that will help rescuers acquire and develop skills, knowledge and methods to mitigate negative effects of occupational stress.

\section{The concept of resilience}

Lately, more and more attention it's given to the concept of resilience, which has a wide applicability. Resilience can be defined as the ability of a system to cope with change, to withstand or to recover from significant threats to its stability, viability or development [3]. Applied to the field of mental and physical health, the concept of resilience refers to the fact that individuals manage to maintain a normal state of physical and mental functioning, despite exposure to sometimes extraordinarily high levels of stress, and to avoid serious mental disorders. This concept emphasizes the person's abilities to survive and even develop as a result of the traumatic events to which one was exposed. The concept is of Latin origin (resilience $=$ rebound from a violent physical shock), and refers to the ability to successfully cope with the worst adversities, disasters, traumatic experiences, falls and the negative effects of stress [4].

In the context of rescue activities, resilience describes the inner strength to face fear and the diversity of actions that may occur during interventions. Resilience is based on the ability to mentally, emotionally, behaviourally and spiritually adapt and combines physical, mental and social skills to generate optimal performance. Training for development of resilience is specific to each of its components. The objectives of developing resilience, are presented in fig. 1. 


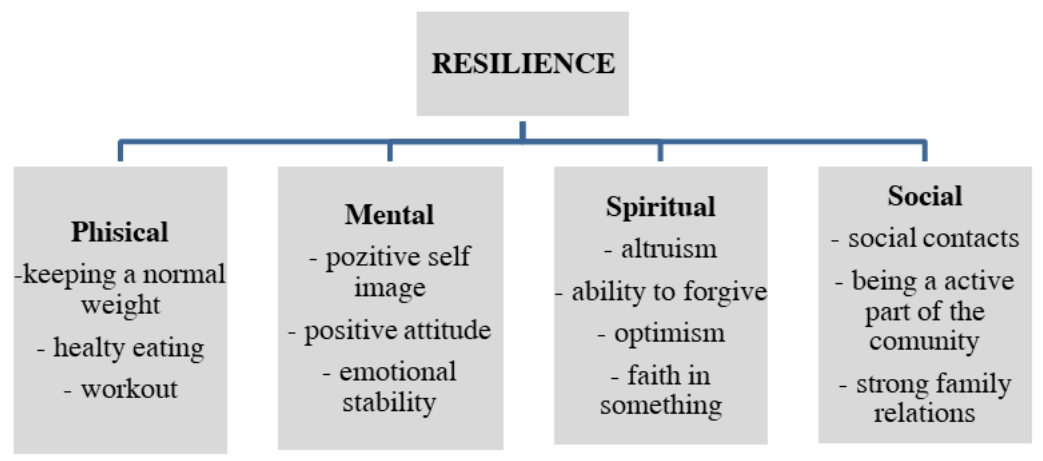

Fig. 1. Resilience objectives [5]

A person's resilience is essential to reduce the effects of stress. Each person responds differently to stressful events depending, also, on their resilience factors. It is important for people to identify their own protective factors against stress and to learn how to develop new skills to deal with the unpleasant situations they encounter. Intervention and rescue personnel are obliged to perform a behaviour completely appropriate to the task, as a condition of achieving the proposed objectives. Regulation and self-regulation of mental states thus becomes, at the same time, both a means for obtaining performance and an objective of the training process $[6,7]$.

Numerous factors contribute to the development and modulation of resilience, among which are genetic, epigenetic, developmental factors, psychosocial, neurochemical factors and certain specific neurological circuits [6]:

- Genetic factors: a series of genes correlated with the neuropeptide Y, with the hypothalamic-pituitary-cortico-adrenal axis, with the noradrenergic, dopaminergic and serotonergic systems as well as with genetic polymorphs significantly contribute to trauma and stress responses.

- Epigenetic factors: refers to functional changes in the genome, without changes in the DNA sequence. Epigenetic differences may be a consequence of exposure to stress-related factors during critical periods of development, and therefore contribute to susceptibility to certain psychiatric disorders (e.g., depression, drug addiction and schizophrenia).

- Factors correlated with the development process, e.g.: prenatal stress, childhood experiences, chronic psychological and social stress, the degree of perceived control over the stressor, "learned helplessness", etc.

- Psychosocial factors: individual characteristics and behaviours, certain cognitive processes, personality traits and active coping mechanisms. In this sense, cognitive-behavioural psychological intervention techniques are increasingly recognized as effective methods for development of resilience, involving both the development of functional coping mechanisms and management of dysfunctional thoughts and emotions, learning relaxation techniques to reduce or overcome stress.

- Neurochemical factors: these factors interact and influence each other, producing effects of regulating the process of short- and long-term adaptation to stress (serotonin, norepinephrine, neural circuits, etc.).

\section{Prototypical trajectories following exposure to potential trauma}

Resilience can be conceived as one of the ends of the continuum from vulnerability to emotional dysfunction and pathology, as a result of exposure to stressful experiences. Recent studies have shown that resilience and healing are distinct and empirically separable 
outcomes of trajectories following traumatic events. Bonanno [8] presents the following prototypical trajectories that occur as a result of exposure to potentially traumatic events (fig. 2):

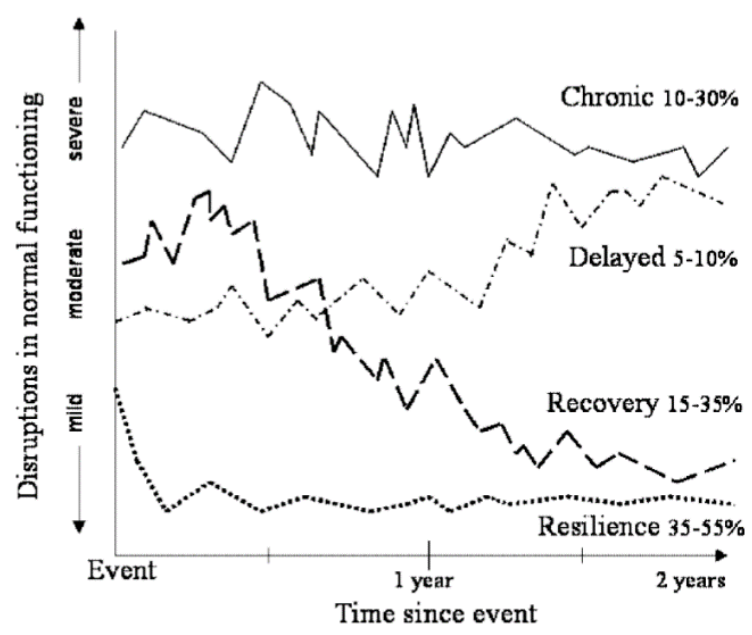

Fig. 2. Prototypical trajectories of disruption in normal functioning during the 2 -year period following a loss or potential trauma [8]

- resilience, which occurs in $35-55 \%$ of cases. Resilience is characterized by lowintensity and short period disturbances. Resilient individuals may initially experience a brief but relatively intense moment of distress or may struggle for a short period of time to maintain mental balance (e.g., a few weeks of sporadic difficulty concentrating, intermittent insomnia, daily well-being variability etc.).

- recovery, which occurs in $15-35 \%$ of cases. Healing is characterized by moderate to severe initial symptoms, which interrupt normal functioning, followed by a gradual decrease in symptoms over several months, before returning to pre-traumatic levels.

- delayed reaction, which occurs in $5-10 \%$ of cases. Delayed reaction is characterized by moderate to mild initial symptoms, which interrupt normal functioning, followed by their worsening, reaching severe levels.

- chronic reaction, in $10-30 \%$ of cases. Chronic reaction is characterized by the occurrence of severe symptoms that disrupt normal functioning and which, although slightly fluctuating, persist on the long term.

In turn, Lepore and Revenson [6] delineated the resilience of restoration, resilience, and reconfiguration. In recovery or healing, emphasis falls on elasticity of response: people are affected, but do not give into the stressor and, finally, return to their initial state. In resistance, the person's functioning is not seriously affected by the stressor, and if it is affected, the effect is relatively minor, as in some cases of loss - perceived as a natural part of life, but not in cases of major losses. Finally, the term reconfiguration refers to the idea of a permanent alteration and adaptation to consequences of trauma.

\section{Strategies to increase psychological resilience}

Development of resilience should focus on amplifying existing strengths (physical and mental characteristics and abilities), rather than managing the negative effects of operational stressors. Therefore, in intervention and rescue actions, resilience involves development of courage, the power to cope with the fear of injury or loss of life, responding to acts of 
aggression and surviving. To develop mental, spiritual and social components, a strong physical component is needed. This involves developing strategies for training and maintaining good health and fitness: ensuring proper nutrition and a regular eating schedule; maintaining health and reducing substance use; physical endurance training, through special physical training programs; sleep control, avoiding consumption of energizers that disrupt the normal sleep-wake cycle $[9,10]$.

Increasing resistance to stress is best to be achieved before intervention, through a psychological training program, able to help intervention and rescue personnel to cope with the emotional burden that characterizes their activity. Resilience training means the acquisition of skills that will help individuals deal with stressful situations, without developing dysfunctional reactions and behaviours. The idea of strategies to increase psychological resilience relies on the fact that, while some people have the ability to naturally reach a certain level of resilience, others can learn different behaviours to help them deal with difficult situations.

Possible measures include:

a. Stimulating the internal control locus for intervention personnel could help them to carry out their tasks without further affecting their mental health. Self-esteem, selfconfidence and self-image are all factors that impact how an individual manages stress reactions during and after a traumatic event.

Increasing self-efficacy (fig. 3) in a particular area is recommended as a precursor to increasing performance in that area and the main means by which self-efficacy can be increased are:

- references to previous performances (previous success), which creates the feeling of power [11];

- vicarious learning or exposure to behaviours and possibilities of others;

- verbal persuasion from significant others (e.g., encouragement of formal or informal leaders);

- psychophysiological regulation in the moment of anticipating / carrying out a task (emotions, mood, stress or physical reactions can influence the way we perceive our own abilities).

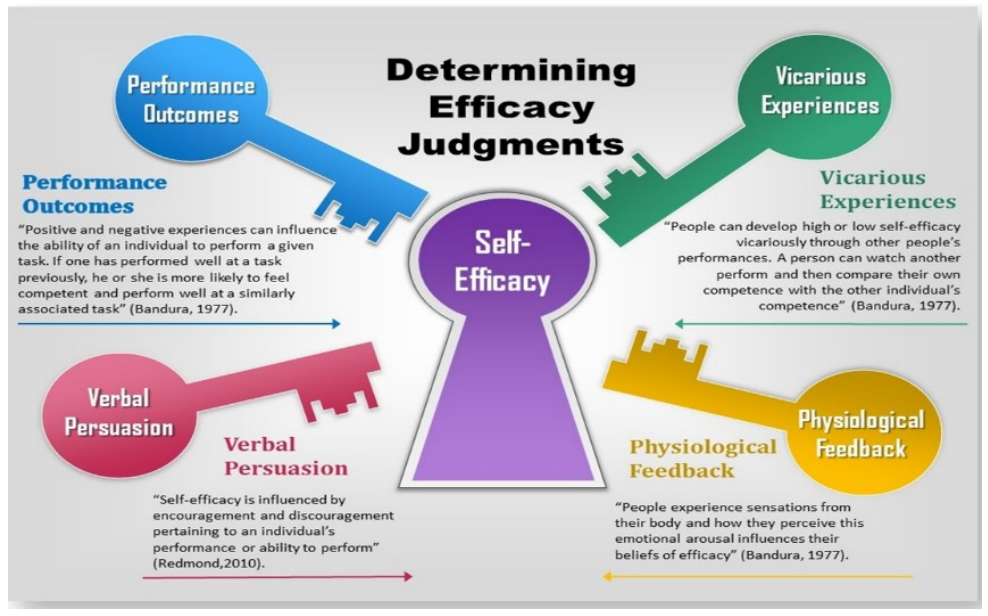

Fig. 3. Keys to raising self-efficacy [12]

References to previous performance, verbal persuasion but to some extent and vicarious learning are methods that can best be implemented by leaders of an intervention team. In 
order to reduce and confute the action of stress by increasing self-efficacy, team leaders can intervene, both individually and in groups [13].

b. Experiencing feelings of confidence and security can act as a buffer for negative stress reactions. Social support from family and colleagues and a well-developed personal support network can help intervention and rescue personnel get over traumatic events more easily.

c. Well-coordinated teamwork, with the support of colleagues, provides a sense of security and belonging, reducing mental and emotional strain at the event. Since experienced staff appear to be less at risk for mental health problems, teams of personal with varying degrees of experience could provide increased social support for the less experienced.

d. Enhancing robustness. Individuals who have high levels of personality robustness are less sensitive to workplace stressors and more able to adapt and cope with new stressors compared to those with low levels of personality robustness. Robustness can be increased in the following ways [14]:

- realistic training: setting adequate expectations, developing coping skills and stress management;

- development of positive thinking;

- developing group cohesion and social support, in this sense an important role being played by leaders of rescue teams;

Interventions to increase or strengthen robustness are part of the same field of personal development, as well as interventions to increase self-esteem and self-efficacy.

e. Enhancing self-esteem. An overall assessment of one's self in positive terms does not necessarily equate to mental health or functional behavioural consequences. It is advisable for the self-esteem to have a medium level of development, because a very low self-esteem, as well as a very high self-esteem can lead to psychological problems.

\section{Conclusions}

- Organizational environments have always been receptive to what psychologists call individual differences, such as asymmetries that underlie differences between two or more people.

- A person's resilience is essential to reduce the effects of stress.

- Numerous factors contribute to the development and modulation of resilience, among which are genetic, epigenetic, developmental factors, psychosocial, neurochemical factors and certain specific neurological circuits.

- Resilience is a different result from recovery, which appears as a different trajectory following exposure to potentially traumatic events.

- Resilience is the most common result of exposure to potentially traumatic stimuli, followed by gradual resolution and recovery.

- There are numerous and sometimes unexpected factors that can ensure the emergence of resilience, such as supportive relationships and individual factors, such as adaptability and flexibility to change.

- Improving psychological characteristics, such as the feeling of coherence, resilience and the ability to psychologically adapt, can facilitate coping with difficult, traumatic situations. The feeling of coherence is related to intelligibility (perceiving the world as easy to understand), flexibility (taking into account all the appropriate and efficient resources available to manage or control life events effectively) and meaning (feeling that it makes sense to care about what happens, that it is really worth the effort and it is satisfying). 
Robustness defines people with a high level of commitment, control, responsibility, who treat challenges as opportunities for personal development.

- In workplaces these characteristics can be improved through team cohesion, collective identity, common values, good leadership, trust and mission objectives (goal orientation).

Through the current paper we managed to highlight the important role played by the development of resilience in mitigating the effects of stress and in developing a methodology, implementable at organizational level, in order to manage occupational stress associated with intervention and rescue activities in toxic or explosive environments.

\section{References}

1. C. Mairean, M. N. Turliuc, Psychology of trauma, Polirom Publishing House, Iaşi, (2014)

2. J. E., Maddux, (Ed.), Self-efficacy, adaptation, and adjustment: Theory, research, and application., Springer Science \& Business Media, (2013)

3. S. Parsons, A.-W. Kruijt, E. Fox, A Cognitive Model of Psychological Resilience, Journal of Experimental Psychopathology., Volume: 7 issue: 3, page(s): 296-310, (2016)

4. D. Alexander, Resilience and disaster risk reduction: an etymological journey. Natural Hazards and Earth System Sciences, 13, (2013)

5. G.A. Bonanno, Loss, trauma, and human resilience: Have we underestimated the human capacity to thrive after extremely aversive events., American Psychologist, 59, 20-28, (2004)

6. C. Demian, Resilience and the factors that influence it. Practical implications for children, adolescents and adults, Journal of School and University Medicine, IV, Nr. 1, pg. 40- 47, (2017)

7. Z. Bogathi et al., Organisational and work psychology handbook, Polirom Publishing House, Bucharest, (2004)

8. G.A. Bonanno, Resilience in the Face of Potential Trauma, Current Directions in Psychological Science, 14(3), (2005)

9. S. J. Lepore, T. A Revenson, Resilience and Posttraumatic Growth: Recovery, Resistance, and Reconfiguration. In L. G. Calhoun \& R. G. Tedeschi (Eds.), Handbook of posttraumatic growth: Research \& practice (pp. 24-46). Lawrence Erlbaum Associates Publishers, (2006)

10. S. S Luthar, E. J Crossman, P. J. Small, Resilience and adversity. In R.M. Lerner and M. E. Lamb (Eds.). Handbook of Child Psychology and Developmental Science (7th Edition, Vol. III, pp. 247-286). New York: Wiley, (2015)

11. S. Beattie, T. Woodman, M. Fakehy, C. Dempsey, The role of performance feedback on the self-efficacy-performance relationship., Sport, Exercise, and Performance Psychology, 5(1), $1,(2016)$

12. https://www.simplypsychology.org/self-efficacy.html

13. V. Mărineanu, Manual for psychological training and control of operational stress, Army Editorial Technical College Publishing house, Bucharest, (2015)

14. Kovacs, G. A. Găman, D. Pupăzan, C. Ilie, A. Gireadă, Psychological training program for intervention and rescue personnel in toxic / flammable / explosive environments, $8^{\text {th }}$ International Occupational Health and Safety Symposium-SESAM, Proceedings of the Symposium, vol II, ISSN 1843-6226, (2017) 